\title{
Lithium acne
}

\author{
Federica Scarfi MD, Meena Arunachalam MD
}

A 40-year-old woman presented with a severe eruption of cysts, papules, nodules and a few comedones on her face (Figure 1). She had no other lesions on her body. She had no history of acne or other dermatologic conditions. She had started taking lithium carbonate 4 months earlier for the treatment of bipolar disorder; the outbreak began during this period. The results of a complete blood count and metabolic panel were normal, as were her levels of sex hormones and serum lithium. A skin biopsy showed follicular obstruction and cystic dilatation of the pilosebaceous duct (Figure 1). We diagnosed lithiumrelated acne and recommended that lithium be replaced by an alternative drug. At her 6-month follow-up visit, the acneiform eruption had improved, and by 9 months it had completely resolved.

Estimates of the incidence of lithium-related skin conditions range from $3 \%$ to $45 \%$. $^{1}$ In addition to acne, associated conditions include psoriasis, eczema, hair loss, hidradenitis suppurativa, nail dystrophy and mucosal lesions. ${ }^{1}$ Acneiform eruptions tend to occur within the first 6 months of therapy, and they may be linked to lithium's tendency to increase circulating neutrophil chemotaxis, stimulate lysosomal enzyme release, and induce follicular hyperkeratosis. ${ }^{1-3}$ The typical clinical presentation is a monomorphic, papulopustular eruption of the trunk and extremities, without the presence of comedones or cysts. ${ }^{1,2}$ Our patient's case is atypical because of the presence of comedones and the facial distribution. Although unusual, this presentation has been previously reported..$^{2,4}$ Lithium-related acne may be resistant to conventional treatments, and a decrease or discontinuation of the medication can sometimes be beneficial, as it was here. ${ }^{1-4}$

\section{References}

1. Yeung CK, Chan HH. Cutaneous adverse effects of lithium: epidemiology and management. Am J Clin Dermatol 2004;5:3-8.

2. Du-Thanh A, Kluger N, Bensalleh H, et al. Drug-induced acneiform eruption. Am J Clin Dermatol 2011;12:233-45.

3. Gupta AK, Knowles SR, Gupta MA, et al. Lithium therapy associated with hidradenitis suppurativa: case report and a review of the dermatologic side effects of lithium. J Am Acad Dermatol 1995;32:382-6.

4. Oztas P, Aksakal AB, Oztas MO, et al. Severe acne with lithium. Ann Pharmacother 2001;35:961-2.

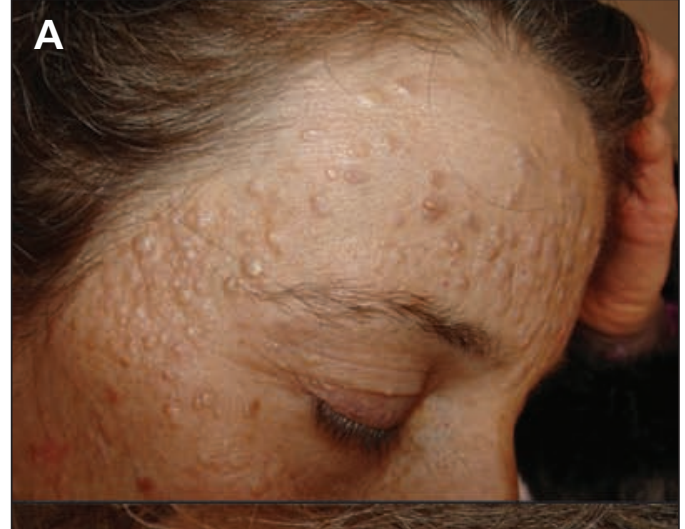

Competing interests: None declared.

This article has been peer reviewed.

Affiliation: Department of Critical Care Medicine and Surgery, Division of Dermatology, University of Florence, Florence, Italy

Correspondence to:

Federica Scarfi, scarfif@libero.it

CMAJ 2013. DOI:10.1503 /cmaj.130009 